Lexis Vol. XLV (1) 2021: 125-162

\title{
Anglicismos en la prensa digital regional canaria: Análisis del periódico La Provincia*
}

\author{
Carmen Luján-García \\ https://orcid.org/0000-0001-7050-777X \\ Universidad de Las Palmas de Gran Canaria \\ carmen.lujan@ulpgc.es
}

\begin{abstract}
RESUMEN
Numerosos estudios han documentado una notable presencia de anglicismos en múltiples áreas de la vida cotidiana de los hispanohablantes. Este trabajo demuestra, con datos actuales, la penetración del inglés a través de la prensa digital. Se ha analizado la presencia del inglés en la versión digital del periódico canario La Provincia (España) durante el primer trimestre de 2019, con el objetivo de demostrar cómo cualquier lector y hablante de a pie está expuesto a anglicismos diariamente. El método, basado en la lectura diaria y extracción manual de los anglicismos hallados en todas las secciones del periódico, revela que el tipo de anglicismo más frecuente es el crudo o no adaptado. A partir de dicho método, además, se analizan los marcadores ortotipográficos empleados y se discuten las funciones de estos anglicismos en la prensa analizada.
\end{abstract}

Palabras clave: Anglicismos, no adaptados, prensa digital, Canarias

\footnotetext{
* Este trabajo se enmarca dentro del proyecto de investigación Canarias y América. Intercambio lingüístico y cultural. El impacto de la cultura anglonorteamericana en la identidad de los canarios a través de los medios de comunicación escritos y orales, financiado por el Gobierno de Canarias a través de la Universidad de Las Palmas de Gran Canaria (CEI 2018-32).
}

https://doi.org/10.18800/lexis.202101.003 
Anglicisms in the Canarian Regional Digital Press: An Analysis of the Newspaper La Provincia

\begin{abstract}
A number of studies have documented the remarkable presence of Anglicisms in various areas of Spanish speakers' daily life. This paper reports, with updated data, the penetration of English through the digital press. The presence of English has been examined in the digital edition of Canarian newspaper La Provincia (Spain) during the first term of 2019 aiming at showing how any reader and speaker is daily exposed to Anglicisms. The method, based on the manual reading and extraction of Anglicisms in all the sections of the newspaper, reveals that the most frequently used kind of Anglicism is the non-adapted one. Furthermore, using said method, the spelling markers and, finally, the functions of these Anglicisms in the analyzed press are discussed.
\end{abstract}

Palabras clave: Anglicisms, non-adapted, digital press, Canary Islands

\title{
1. Introducción
}

Está fuera de toda duda la notable presencia de la lengua inglesa en múltiples áreas de nuestra vida diaria. Así lo corroboran numerosos estudios que han analizado dicha presencia de anglicismos en la prensa en español en múltiples y variados campos. Haremos referencia a algunos de aquellos trabajos más recientes dentro de los dominios más proclives a la penetración de unidades léxicas anglicadas. Algunos de estos terrenos son el del deporte, con trabajos que analizan la prensa deportiva (Rodríguez González 2012, 2016; Campos-Pardillos 2015; Rodríguez-Medina 2016, 2021; Vázquez Amador y Lario de Oñate 2015); la moda, la belleza y los famosos (Balteiro y Campos 2012, Luján-García 2017a; Tejedor Martínez 2017, 2021; Vega Falcón 2017); el dominio de la informática y los nuevos medios de comunicación (Pano 2007, Bolaños-Medina y Luján-García 2010, Luján-García 2017b, Luján-García, 2021), así como la prensa económica, el comercio y las finanzas (Vélez Barreiro 2003, López-Zurita 2005). 
Otros campos, tal vez menos explorados y que resultan más curiosos, también han sido objeto de investigación por parte de diversos autores. Un ejemplo de ello es el de la prensa rosa (Vázquez Amador 2018). Además, se ha explorado el empleo de anglicismos en el campo de la sexualidad como herramienta atenuadora para evitar el término tabú o malsonante en español (Crespo-Fernández y Luján-García 2013, 2017, 2018), y es destacable también el estudio del anglicismo en el terreno de los nombres de los juguetes y juegos dirigidos a los más jóvenes (Luján-García 2011, 2015).

Algunos trabajos se centran en el análisis reciente de anglicismos en la prensa de lugares concretos; entre ellos podemos destacar estudios como el de Raidel Naranjo (2017) que analiza la prensa de Santiago de Cuba. Otras publicaciones han examinado aspectos como los procesos de formación de neologismos en la prensa española (Esteban Asencio 2008). También el análisis de los neologismos usados por periodistas ha sido examinado (Gerding, Cañete y Adam 2018).

Todas estas publicaciones tienen el denominador común de que ponen de manifiesto la notable y creciente presencia de anglicismos, bien sean no adaptados, adaptados, híbridos o falsos anglicismos en la prensa y, pese a centrarse en un único periódico regional de Canarias, con casi total seguridad se podrían extrapolar los resultados a la prensa de casi cualquier lugar de habla hispana. Esto nos permite resaltar que la prensa, ya sea en formato papel o digital, constituye una importante puerta de entrada para la penetración y más o menos libre circulación de anglicismos en el español. Cabe destacar que un estudio previo realizado hace varias décadas examinó la presencia de anglicismos en el mismo periódico canario en formato papel (Luján-García 1999), pero, sin duda, el empleo de unidades léxicas anglicadas se ha visto incrementado notablemente, como se verá más adelante. De cualquier forma, la razón que justifica este estudio es que muchos de estos trabajos se han centrado en la prensa y no en la digital, que parece extenderse cada vez más entre los lectores. Además de ello, se debe señalar que algunos de 
estos trabajos ya cuentan con algunos años, por lo que se pretende aportar datos actuales.

La hipótesis de partida de este estudio pretende poner de manifiesto el incremento del impacto de la lengua inglesa en la lengua española focalizándose en el lenguaje periodístico español, que parece estar cada vez más plagado de anglicismos en las distintas secciones de la prensa analizada. Para ello se contrastarán los resultados de este estudio con el realizado por Luján-García en 1999. Esta hipótesis, además, parte de la base de que la mayor presencia de anglicismos es la de los crudos o no adaptados al español y de que muchos de los anglicismos empleados actualmente no se usaban hace dos décadas, mientras que buena parte de los que se empleaban en 1999 ya han sido incorporados y normalizados por el Diccionario de la Lengua Española $(D L E)$ de ahora en adelante.

\section{Objetivos}

En este trabajo pretendemos revelar el grado de presencia que la lengua inglesa tiene en la prensa digital publicada en Canarias. Concretamente, nos centraremos en el análisis del periódico regional $L a$ Provincia editado y leído en el archipiélago canario. Con tal fin, las siguientes preguntas de investigación se han formulado:

- ¿Qué tipo de anglicismos es el más frecuente en el análisis de la edición digital del periódico La Provincia?

- ¿Qué funciones cumplen estos anglicismos en la prensa analizada?

- ¿Qué marcadores ortotipográficos son mayormente empleados para señalar el empleo de anglicismos por parte de los periodistas o redactores de noticias?

\section{Método}

El método empleado se basó en el seguimiento diario y extracción de anglicismos de la edición digital publicada en la web del propio periódico (https://www.laprovincia.es/). La Provincia es un diario 
matutino con una tirada de 25.077 ejemplares en papel (media de 2012), perteneciente al grupo editorial Prensa Ibérica creado en 2008. La razón por la que se eligió la versión digital abierta y no la edición en papel se debe a que esta primera es gratuita, a diferencia también de la edición digital más extendida que requiere de subscripción y pago al igual que la versión en papel y que, por lo tanto, limita y no es accesible a cualquier lector.

El seguimiento de las noticias publicadas se realizó mediante la lectura diaria de estas en la edición digital abierta y gratuita a lo largo del periodo del 1 de marzo hasta el 31 de mayo de 2019 (ambos inclusive). Tras leer los titulares y noticias, se extrajeron los anglicismos léxicos presentes, así como las oraciones empleadas dentro de sus correspondientes artículos. Asimismo, se recopiló el día, mes, tipo de anglicismo y si es que este tenía alguna marca ortotipográfica. Se descartaron aquellos anglicismos que ya están recogidos en el DLE, aunque sí se contemplaron algunos casos que, pese a que están recogidos en el DLE con algún tipo de adaptación, aparecen usados en el corpus compilado sin adaptación, con su forma original inglesa.

Con respecto a la categorización de anglicismos, se empleará la más reciente publicada por Pulcini, Furiassi y Rodríguez González (2012) en la que se distinguen los préstamos directos de los indirectos. Los anglicismos léxicos directos pueden ser de tres tipos: préstamos adaptados y no adaptados; falsos anglicismos o pseudoanglicismos y anglicismos híbridos. Estos tipos serán el objeto de análisis en el presente estudio.

\section{Resultados}

En esta sección se realizará un análisis tanto cuantitativo como cualitativo de los resultados, como se refleja en los próximos epígrafes. 
3.1. Tipos de anglicismos

Tabla 1. Desglose de anglicismos por distintas categorías

\begin{tabular}{cccccc}
\hline & $\begin{array}{c}\text { NO } \\
\text { ADAPTADOS }\end{array}$ & ADAPTADOS & HÍBRIDOS & $\begin{array}{c}\text { FALSOS } \\
\text { ANGLICISMOS }\end{array}$ & TOTAL \\
\hline ANGLICISMOS & 293 & 23 & 8 & 4 & 328 \\
\hline$\%$ & $89,3 \%$ & $7 \%$ & $2,4 \%$ & $1,2 \%$ & \\
\hline
\end{tabular}

Como se puede observar en la tabla 1, el porcentaje mayor, con una diferencia notable, es el de anglicismos sin ningún tipo de adaptación. Estos préstamos son usados en el español tal como vienen del inglés, sin experimentar ningún tipo de adaptación. Otros estudios recientes (Rodríguez Díaz 2011; García-Morales, González-Cruz, Luján-García y Rodríguez-Medina 2016) han revelado que también en el campo de la publicidad televisiva ocurre algo similar, es decir, se emplean numerosos anglicismos y la mayoría de ellos no emplean ningún tipo de adaptación: se usan en su forma pura del inglés.

\subsubsection{Anglicismos No Adaptados $=293$ casos}

Son unidades léxicas que se integran en el español tal cual provienen del inglés. Pulcini, Furiassi y Rodríguez González los definen como:

a word or multi-word unit borrowed from the English language without or with minor formal and semantic integration, so that it remains recognizably English in the $R L$. To a greater or lesser extent, phonological integration always takes place because of differences between the sound systems of individual languages (2012: 6).

Fitness o airbag podrían ser algunos ejemplos de este tipo de préstamos en español.

En este epígrafe se desglosará el corpus de anglicismos no adaptados por orden alfabético, considerando no solo el tipo de anglicismo, sino también el uso de algunos de estos extranjerismos en contexto. Por limitaciones de espacio, no es posible incluir ejemplos en contexto de todas las unidades léxicas; no obstante, en el caso de 
algunos anglicismos menos frecuentes o extendidos se ha incluido una definición o explicación del término.

Letra $\mathrm{A}=6$ casos

App, animal print, anti-frizz, after sun, adware, airless.

Anti frizz hace referencia a un tratamiento capilar antiencrespamiento.

(1) Decía que la plancha es un buen arma anti-frizz, pero no solo eso ( $\mathrm{La}$ Provincia, 08/03/2019).

Adware se emplea en informática para referirse a "un tipo de software que, de modo automático, exhibe al usuario anuncios publicitarios"1.

(2) La aparición de anuncios emergentes nada más desbloquear el «smartphone es un claro anuncio de infección, en este caso, de un <adware (La Provincia, 10/04/2019).

Airless se refiere a una tecnología que permite la pulverización de pintura y otros materiales a alta presión ${ }^{2}$.

(3) El envase airless (La Provincia, 26/04/2019).

Letra $\mathrm{B}=26$

Baby blond, baby lights, baby shower, batch cooking, beauty, beauty freaks, beauty victim, beauty look, bed and breakfast, big data, big player, biker, bill board, biopic, bluetooth, blush, board, body milk, broker, bronzer, buggy, burn out, bodyboard, bullying, business intelligence, back-up.

Batch cooking consiste en cocinar un día la comida de toda la semana con el objeto de comer sano, pese a no tener tiempo de cocinar.

(4) ¿Has oído hablar del batch cooking? (La Provincia, 14/03/2019).

En https://definicion.de/adware/ [Consulta: 18/06/2020].

2 En https://www.airless-discounter.de/ [Consulta: 19/06/2020]. 
Business intelligence es la habilidad para transformar los datos en información, y la información en conocimiento, para optimizar la toma de decisiones en los negocios ${ }^{3}$.

(5) Son expertos en la implantación de software de gestión para pequeñas y medianas empresas, de todos los sectores, para mejorar el funcionamiento y gestión de su negocio, con el objetivo de dar la mejor respuesta y servicios a sus clientes, en la asesoría, y mantenimiento tanto de las áreas de infraestructuras de IT, como en sus soluciones de software ERP, CRM o Business Intelligence, entre otras (La Provincia, 30/04/2019).

$\mathrm{C}=32$

Call center, casual, celebrity, chat, checkpoint, cheer, cheerleader, chill out, chips, chunky sneakers, cyberbullying, city, class, click, cloud computing, clown, coach, coaching, cocktail, common rail, contouring, cookies, cool, cowboy, coworking, crop top, cross, crossfit, curvy, cushion, customer experience, cut out.

Coworking es una filosofía de trabajo y de vida que permite a profesionales de diferentes sectores, compartir un mismo espacio de trabajo sin perder su independencia ${ }^{4}$.

(6) Ocurrió en el centro de coworking de la calle Bolivia, número 11, en el barrio de Guanarteme (La Provincia, 24/04/2019).

Crop top es un top corto.

(7) El primer look se trata de una falda de corte flamenco pero con una amplia apertura lateral y un crop top en blanco (La Provincia, 16/04/2019).

Letra $\mathrm{D}=10$

Dad sandals, dermaroller, DIY, DJ, drag, drag finalist, drag queen, drag race, dry brushing, dummy.

\footnotetext{
En https://www.isotools.org/2018/03/15/ [Consulta: 19/06/2020].

4 En https://mentorday.es/diccionario-emprendedores/letra/coworking/ [Consulta: $19 / 06 / 2020]$.
} 
Dad sandals alude a un tipo de sandalia con suela de corcho que estuvo muy de moda en los sesenta y han vuelto al panorama de la moda recientemente.

(8) son conocidas como 'dad sandals', o también por su versión en castellano, zapachanclas, y se encuentran ya en los escaparates de las grandes tiendas de ropa 'low cost' (La Provincia, 06/05/2019).

Dermaroller, pese a que no aparece recogido en el Oxford English Dictionary (OED de ahora en adelante) o el Cambridge English Dictionary (CED a partir de ahora), parece ser usado tanto en España como en Gran Bretaña y se refiere a un pequeño aparato de fácil manejo que en su extremo tiene un rodillo provisto de diminutas agujas de titanio. Deslizado sobre la piel realiza una estimulación mecánica que mejora microlesiones 5 .

(9) Cómo se usa un dermaroller (La Provincia, 01/03/2019).

Dummy es un maniquí que imita o suple a una persona.

(10) Durante la prueba de choque frontal, con el dummy que simula a un niño de 1 año y medio, la silla Babystyle Oyster + Base Duofix Isize se desprende de la base y sale proyectada hacia adelante, lo que expone al niño a un riesgo de daños serios (La Provincia, 07/04/2019).

Letra $\mathrm{E}=7$

E-commerce, economy class, e-mail, escape rooms, expertise, express, eyeliner.

Escape room es un juego que consiste en usar la lógica y el ingenio para escapar de una sala antes de 60 minutos. Para ello se aportan al jugador acertijos y enigmas.

(11) De videojuegos a escape rooms, las empresas cada vez incorporan más la gamificación o mecánicas de juego para seleccionar a su personal (La Provincia, 11/05/2019).

Eyeliner es un delineador de ojos.

5 En https://www.facebook.com/genesiswellness.com.ar/posts/2816348895049585 [Consulta: 19/06/2020]. 
(12) Sombras, eyeliners y máscaras de pestañas se tiñen de colores puros para recibir a la primavera (La Provincia, 03/04/2019).

Letra $\mathrm{F}=16$

Fake news, fashion week, fast ferry, feedback, filler, film, fingers, firmware, first class, fitness, flick, flow, freak, frizz, full time, fulldome.

Filler, en el contexto de la estética, alude a sustancias que se emplean para rellenar las arrugas de la piel.

(13) El ácido hialurónico de los fillers es diferente también al que viene en los sueros que compramos en la tienda (La Provincia, 05/04/2019).

Fingers son los dispositivos que se emplean en aeropuertos para conectar la terminal con el propio avión, permitiendo a los pasajeros un acceso cómodo a la aeronave.

(14) Problemas con las maletas, con los planes de vuelo (los pilotos no pueden operar hasta tenerlos en la mano) y hasta retrasos con la colocación de los fingers serán algunos de los efectos más visibles que soportarán los usuarios de la red de AENA (La Provincia, 11/04/2019).

Firmware es un software que maneja físicamente un hardware.

(15) También se podrá modificar el 'firmware' para realizar "labores de mantenimiento del dispositivo o sistema con el objetivo de hacer que funcionen según sus especificaciones originales" (La Provincia, 08/05/2019).

Fulldome es un formato de proyección inmersivo basado en películas panorámicas en $360^{\circ}$ dentro de una estructura de domo ${ }^{6}$.

(16) Unas proyecciones, nueve en total, que permitirán ver las imágenes en 360 grados, una experiencia novedosa en una suerte de lienzo digital con un gran potencial creativo, que brinda al espectador la oportunidad de estar en un espacio sin límites y ser un descubridor, gracias al sistema de proyección fulldome, aclara Marta Hernández, concejala de Cultura (La Provincia, 12/04/2019).

6 En https://proyectoidis.org/fulldome/ [Consulta: 19/06/2020]. 


\section{Letra $\mathrm{G}=9$}

Gadget, grappling, guy, gaming, gamer, gentleman, glitter, glow, green fee.

Grappling procede de la palabra inglesa grip y se refiere a una modalidad de lucha que busca tener bajo control al oponente sin efectuar ningún golpe, para después ganar el combate ${ }^{7}$.

(17) La alcaldesa y el concejal de Deportes agasajan a los miembros de este colectivo, con varios campeones de Canarias y de España de grappling (La Provincia, 07/03/2019).

Un estilo gentleman se refiere a una prenda de ropa con un estilo elegante y cuidado y modales muy finos por parte de quien vista la prenda.

(18) Algunas de las que triunfan son rockabilly, militar o estilo gentleman (La Provincia, 04/04/2019).

Letra $\mathrm{H}=10$

Hackathon, balter, handling, hashtag, hat-tricks, highlighter, bighlights, hipster, hit, hosting.

Halter es una cinta en la que el cuerpo de un vestido sin mangas o la parte superior se fija o se mantiene detrás del cuello, dejando los hombros y la espalda desnuda.

(19) existen en el mercado múltiples diseños que se adaptan al cuerpo de cada mujer y realzan la figura: braguitas altas para alargar las piernas, sujetadores con escote corazón o halter para dar volumen a un pecho pequeño, bañadores completos para disimular tripita [...] (La Provincia, 17/04/2019).

Hat-trick o triplete se refiere a la acción de marcar tres goles un mismo jugador en un mismo partido.

(20) el killer charrúa con 21 goles y cuatro hat-tricks, se convirtió en el pichichi de la liga al sumar una asistencia más y jugar menos minutos que Willis Plaza, jugador trinitense del Churchill Brothers, con quien terminó empatado a goles (La Provincia, 14/03/2019).

7 En https://www.luchaasturias.es/grappling/ [Consulta: 20/06/2020]. 
Hosting es la acción de hospedar o alojar a alguien o bien hacer de anfitrión en un evento.

(21) es un hotel moderno, eficiente y tecnológico, con un excelente "Value4money", especializado en "Hosting" que ayuda y guía a los clientes que lo desean, a organizar de forma proactiva su estancia y vivir una experiencia urbana (La Provincia, 23/04/2019).

Letra $\mathrm{I}=4$

Indie, indoor, influencers, Internet of Things.

Indoor implica el desarrollo de alguna actividad en el interior de un edificio.

(22) Del mismo modo, se obtuvo el correspondiente mandamiento de entrada y registro para la plantación en este caso de interior o también denominada 'indoor', y se detuvo a esta persona, así como de dos personas más que cuidaban la plantación (La Provincia, 12/04/2019).

\section{Letra $\mathrm{K}=4$}

Keylogger, kid, killer, kitesurf.

Keylogger es un software o bardware que puede interceptar y guardar las pulsaciones realizadas en el teclado de un equipo que haya sido infectado 8 .

(23) En el tipo de ataque denominado 'keylogger', el usuario instala inconscientemente un programa malicioso, conocido como 'keylogger', al acceder a un enlace o descargar un archivo de Internet (La Provincia, 05/03/2019).

Killer es un anglicismo metafórico que, como Rodríguez González sostiene, se usa para referirse "a quien destaca sobremanera en el fútbol, que suele ser un delantero muy efectivo, recibe el nombre de crack o killer" (2016: 37).

(24) el killer charrúa con 21 goles y cuatro hat-tricks, se convirtió en el pichichi de la liga al sumar una asistencia más y jugar menos minutos

8 En https://latam.kaspersky.com/blog/que-es-un-keylogger-2/453/ [Consulta: 20/06/2020]. 
que Willis Plaza, jugador trinitense del Churchill Brothers, con quien terminó empatado a goles (La Provincia, 14/03/2019).

Letra $\mathrm{L}=10$

Lifting, light detection and ranging, lipstick, lobby, local craft beer, look beauty, look twinning, lover boys, low cost, lunch box.

Local Craft Beer se refiere, como se observa en la siguiente cita de prensa, a un tipo de cerveza elaborada de forma natural en una destiladora local, sin procesos industriales.

(25) Esta variedad de Jaira, elaborada según la filosofía "Local Craft Beer" y de forma natural, sin filtrar ni pasteurizar, volverá a comercializarse en el mes de diciembre, con una producción exclusiva y una tirada limitada (La Provincia, 17/03/2019).

Look twinning es una expresión que proviene del inglés twin (gemelo) y consiste en vestir igual que alguien. Algo que, normalmente, la gente intenta evitar y que parece haberse convertido en una tendencia de moda en redes sociales como Instagram.

(26) Shakira y Piqué se suman al 'look twinning', la moda de vestirse igual que la pare (La Provincia, 02/04/2019).

Lover boy es una expresión que hace referencia a un hombre que tiene un gran éxito con las mujeres o mantiene relaciones sexuales con ellas, a veces de forma ilícita. En ocasiones se usa como sinónimo de amante o de gigoló.

(27) La fiscal solicita para dos hombres acusados de "lover boys" seis y siete años de cárcel (La Provincia, 13/03/2019).

Letra $\mathrm{M}=19$

Machine learning, main event, make-up, malware, manspreading, masterclass, mentoring, merchandising, microblading, millennial, mindfulness, minibasket, mix, mobbing, mom jeans, mountain bike, multistakebolder, must y must have.

Machine learning o aprendizaje automatizado es el subcampo de las ciencias de la computación y una rama de la inteligencia arti- 
ficial, cuyo objetivo es desarrollar técnicas que permitan que los ordenadores aprendan'.

(28) El campo de investigación de este año se sitúa en la explotación con técnicas de machine learning para descubrir nuevos planetas usando los datos de las misiones Kepler y TESS (La Provincia, 29/03/2019).

Microblading es una técnica de maquillaje cuyo objetivo es corregir o reconstruir completamente una ceja carente de pelo o ausente, realizando cilios de forma artística, creando un aspecto natural ${ }^{10}$.

(29) ¿Qué son el shading y el microblading de los que hablan? (La Provincia, 16/05/2019).

Mom jeans es un término de argot para los vaqueros de tiro alto que originalmente estaban de moda a finales de los 80 y principios de los $90^{11}$. Actualmente se consideran desfasados y no a la moda.

(30) Ideas para renovar tu armario y aprender a combinar los 'mom jeans', 'paper bag', etc. (La Provincia, 12/04/2019).

Multistakebolder es una metodología empleada en el campo de la economía donde intervienen múltiples participantes o aspectos que son tenidos en cuenta a la hora de analizar cuestiones como la reputación de una empresa en el mercado.

(31) Existe un instrumento de evaluación reputacional lanzado en el año 2000, basado en una metodología 'multistakeholder' y compuesta por seis evaluaciones y veinticinco fuentes de información avalado por el instituto Análisis e Investigación (La Provincia, 01/03/2019).

\section{Letra $\mathrm{N}=5$}

Naked, networking, newsletter, non-transfer, nude.

Naked, del inglés "desnudo", hace referencia, en el contexto del ejemplo extraído, a algo natural, sin demasiados aditivos químicos.

\footnotetext{
En https://es.wikipedia.org/wiki/ [Consulta: 21/06/2020].

10 En https://www.esteticvenus.com/ [Consulta: 21/06/2020].

11 En https://es.wikipedia.org/wiki/Mom_jeans [Consulta: 21/06/2020].
} 
(32) Es el caso de Lush, que vende productos "naked", y en los que envasa usa para ello plástico reciclado con residuos de, entre otros lugares, la isla de plástico que hay en el océano (La Provincia, 17/05/2019).

Non-transfer se emplea con frecuencia para hablar de un tipo de lápiz de labios que es permanente y no transfiere, es decir, no mancha los dientes.

(33) También existen los labiales non-transfer y los efectos tattoo ( $\mathrm{La}$ Provincia, 16/04/2019).

Nude es un anglicismo que también significa “desnudo" y es empleado con frecuencia en el campo de la belleza para aludir a un estilo natural, con tonos muy similares al de la piel.

(34) Los labios quedaron únicamente bien perfilados y con un tono nude muy natural (La Provincia, 14/03/2019).

Letra $\mathrm{O}=6$

Offshore, oil free, old school, online, outfit, oversize.

Offshore significa literalmente "fuera de la costa", pero, en el contexto de los ejemplos compilados, se refiere específicamente al servicio de reparación de buques y plataformas petrolíferas fuera de la costa.

(35) La industria 'offshore' se da cita en La Luz para hablar de gas y renovables (La Provincia, 22/03/2019).

Old school es una expresión que hace referencia al aspecto o la manera de hacer las cosas a la antigua, propia de generaciones pasadas.

(36) Diez barberías “Old School” para visitar iya! (La Provincia, 04/04/2019).

Letra $\mathrm{P}=27$

Pack, packaging, paddlesurf, paper bag, partner, patchwork, peeling, pendrive, performance, phishing, photocall, phubbing, playoff, poke bowl, pole, pony, port center, post, post-it, premier, premium, preppy, pride, prime day, print, promaster, push up.

Paper bag se usa para referirse a modelos de pantalón de talle alto que se ajustan a la cintura gracias a una lazada o cinturón que, al 
ceñirse, provocan que la tela se frunza en la parte superior, creando el mismo efecto que una bolsa de papel cuando se ata ${ }^{12}$.

(37) Pantalon Paper Bag de mujer Fórmula Joven (La Provincia, 08/03/2019).

Phishing se utiliza para referirse a uno de los métodos más utilizados por delincuentes cibernéticos para estafar y obtener información confidencial de forma fraudulenta (contraseña o información sobre tarjetas de crédito) $)^{13}$.

(38) La campaña de la Renta abre la veda a los intentos de 'phishing' ( $L a$ Provincia, 04/04/2019).

Phubbing o combinación de los términos phone y snubbing (desprecio, desaire) consiste en menospreciar a quien nos acompaña al prestar más atención al móvil u otros aparatos electrónicos que a su persona ${ }^{14}$.

El 'phubbing' o como el móvil afecta a nuestras relaciones sociales (La Provincia, 11/05/2019).

Port center o puerto central, hace referencia a puertos de grandes dimensiones y mayor tráfico de barcos de Europa.

(39) La delegación de los Puertos de Las Palmas ha visitado los port centers de Rotterdam y Amberes (La Provincia, 02/03/2019).

Premier hace referencia a la primera ministra británica.

(40) Con esta hoja de ruta, la "premier" pone de manifiesto que sigue confiando en la posibilidad de que su acuerdo salga adelante en Westminster en el plazo de siete días (La Provincia, 13/03/2019).

Push up se usa con frecuencia para referirse a un tipo de sujetador que eleva los pechos.

(41) Top de bikini push up Martina con volantes de Calzedonia: 40,00 euro (La Provincia, 17/04/2019).

\footnotetext{
12 En https://mundoclubhouse.com/2020/12/30/ [Consulta: 20/06/2020]

13 En https://www.abc.es/tecnologia/consultorio/abci-phishing-201805170332 noticia.html [Consulta: 21/06/2020].

14 En https://culturacientifica.utpl.edu.ec/2019/03/ [Consulta: 21/06/2020].
} 


\section{Letra $\mathrm{Q}=2$}

Quad and queer

Quad es un vehículo similar a una moto o un triciclo, aunque con cuatro ruedas.

(42) La ordenanza que regula la circulación de los quads entra ya en vigor (La Provincia, 21/03/2019).

Queer en inglés significa raro, excéntrico y se ha empleado para referirse al colectivo LGBT+, no obstante, lo que empezó siendo un insulto ha pasado a adoptar una definición más neutral y se usa para referirse a todas aquellas personas que no se sienten incluidas en la forma tradicional con la que se definen las relaciones (LGBT, pansexuales, intersexuales, asexuales, poliamorosos, etc $)^{15}$.

(43) Nuestras edades son todas, nos sabemos lesbianas, trans, bisexuales, inter, queer, hetero [...]" (La Provincia, 09/03/2019).

Letra $\mathrm{R}=12$

Ranger, reality, reality show, red carpet, reefers, reset, resort, responsive, retail, router, runner, running.

Un ranger es un guardabosques o persona encargada de proteger una selva o parque natural.

(44) 250 'rangers' en Gran Canaria (La Provincia, 04/04/2019).

Reefers se usa a menudo para hacer referencia a un tipo de contenedor refrigerado o con función de frigorífico.

(45) La compañía afirma en un comunicado estar inmersa en "una importante inversión” para ampliar su flota de contenedores reefers ( $\mathrm{La}$ Provincia, 13/03/2019).

Resort es un complejo turístico, normalmente con una categoría de entre 4 y 5 estrellas y que normalmente se halla en un entorno natural.

(46) El grupo Cordial abre un resort de 17 viviendas de lujo en Playa de Mogán (La Provincia, 18/04/2019).

15 En https://lesbicanarias.es/2011/08/22/ [Consulta: 21/06/2020]. 


\section{Letra $\mathrm{S}=55$}

Sandals, scaled, science show, scratch, screener, shade, shading, shakedown, shooting, shopping, shot gun, shoulder surfing, showroom, size, skate, skateboard, skatepark, skater, skating, skiplagging, sleeping pack, smart, smart cities, smartbike, smartphone, smartship, smartwatch, smoky eye, snack, snorkel, snorkelling, soft, software, speaker, spoiler, sportlovers, spyware, stage, stand, stand by, stand up / stand up paddle, start-ups, stick, stories, streamer, streaming, striking, striptease, styler, styling, subprime, sunset, swag y swap.

Scaled es una categoría alternativa a la open en una competición de crossfit. Se trata de una categoría dirigida a atletas que todavía no dominan con soltura todos los movimientos o no mueven cargas muy pesadas.

(47) Los títulos se quedaron en las Islas. María Socorro ganó en la categoría Scaled femenina (La Provincia, 01/04/2019).

Scratch es un lenguaje de programación especialmente diseñado para que todo el mundo pueda iniciarse en el mundo de la programación. Sirve para crear historias interactivas, juegos y animaciones ${ }^{16}$.

(48) El martes, 9 de abril, entre las 10.00 y las 12.00 y entre las 17.00 y 19.00 horas, se celebrarán 14 Talleres en sincronía: Taller de Educación Relacional, Taller de Scratch (La Provincia, 05/04/2019).

Screeners, en el contexto de nuestra muestra, alude a un tipo de zapatillas deportivas inspiradas en los 70 que Gucci ha lanzado.

(49) Las Screeners, las zapatillas de moda “viejas y sucias" que cuestan casi 700 euros (La Provincia, 26/03/2019).

Shakedown en los rallies es un tramo cronometrado de no más de $7 \mathrm{~km}$, especialmente preparado para que los automóviles puedan probar de forma intensa las modificaciones y ajustes que los mecánicos hayan realizado para la carrera ${ }^{17}$.

16 En https://garajeimagina.com/es/ [Consulta: 21/06/2020].

17 En https://es.wikipedia.org/wiki/Tramo_(rally) [Consulta: 21/06/2020]. 
(50) Mientras que en Santa Catalina, las asistencias trabajan para ultimar detalles y poner los coches a punto de cara afrontar su primer reto con el crono, que tendrá lugar mañana con el tramo de calificación y shakedown (La Provincia, 01/05/2019).

Shooting, en el contexto de la muestra, hace referencia a una sesión fotográfica para presentar una colección de moda.

(51) Cuando la supermodelo Natalia Vodianova leyó esta frase atribuida a Steve Jobs, supo que, más allá de pasarelas, focos y shootings, su figura mediática tenía que servir para algo más (La Provincia, 01/04/2019).

Shot gun en el golf se refiere al inicio de un torneo en el que varios jugadores comienzan simultáneamente desde sus agujeros.

(52) La modalidad del torneo será 'shot gun' y el precio del 'green fee' será de 50 euros. La recaudación conseguida con este evento estará dedicada a proyectos que velen por la salud de los niños ( $L$ a Provincia, 14/05/2019).

Shoulder surfing es una forma de fraude con tarjetas de crédito en la que el perpetrador mira por encima del hombro de la víctima mientras retira dinero de un cajero automático, memoriza los detalles de la tarjeta para luego robar con la misma ${ }^{18}$.

(53) La práctica conocida como «shoulder surfing', es decir, espiar a un usuario cuando está escribiendo sus credenciales, una llamada de teléfono suplantando la identidad de alguien que requiere una contraseña (La Provincia, 05/05/2019).

Skiplagging (saltarse vuelos) es una práctica conocida desde hace tiempo: gracias a esta técnica es posible viajar más barata en avión. En muchos viajes ir de A hacia B es más caro que ir de A hacia C pasando por $\mathrm{B}$, así que los usuarios cogen esa última opción y no utilizan el último vuelo de $\mathrm{B}$ hacia $\mathrm{C}^{19}$.

(54) Las aerolíneas están que trinan con el truco que poco a poco está extendiéndose y a través del cual el cliente consigue ahorrarse bas-

18 En https://educalingo.com/es/ [Consulta: 21/06/2020].

19 En https://www.xataka.com/ [Consulta: 21/06/2020]. 
tante dinero. Concretamente se trata del 'skiplagging' o 'ciudad oculta’ y consiste en lo siguiente [...] (La Provincia, 22/03/2019).

Sleeping pack hace referencia a un tipo de mascarilla de noche proveniente de Corea con una textura muy ligera que se absorbe rápidamente en la piel sin dejar una sensación pegajosa o de pesadez y sin tener que retirarla del rostro a la mañana siguiente.

(55) 8 sleeping packs para usar mientras duermes (La Provincia, $15 / 03 / 2019)$.

Spoiler se usa para referirse al texto que anticipa la trama de una película, un libro u otra obra.

(56) Tarantino pide que no se hagan 'spoilers' de su nueva película en Cannes (La Provincia, 21/03/2019).

Spyware es un programa espía que recopila información de un ordenador y después transmite esta información a una entidad externa sin el conocimiento o el consentimiento del propietario de ese ordenador ${ }^{20}$.

(57) El 'spyware' tuvo capacidad para infectar a teléfonos con sistema operativo Apple (iOS) o de Google (Android) (La Provincia, 14/05/2019).

El anglicismo stand ha sido compilado porque, en el ejemplo obtenido, aparece bajo su forma original inglesa y no con la adaptación ortográfica que recoge el DLE: estand. La definición que aporta el DLE sobre el término en cuestión es la de "instalación dentro de un mercado o feria, para la exposición o venta de productos”.

(58) "nos estamos apuntando a todas las ferias, concursos y demás actividades que se están celebrando por este y otros municipios para conseguir poco a poco llegar a nuestro objetivo; también hemos puesto stands para vender nuestro merchandising", afirma (La Provincia, 21/04/2019).

20 En https://es.wikipedia.org/wiki/ [Consulta: 21/06/2020.]. 
Una start-up es una compañía emergente o incipiente, de reciente creación, normalmente fundada por un emprendedor o varios, sobre una base tecnológica e innovadora ${ }^{21}$.

(59) La startup confía en cerrar la operación el 15 de abril y, a partir de entonces, comenzar a operar en tres meses (La Provincia, 15/03/2019).

Striking se emplea en el boxeo para referirse a golpear el saco de entrenamiento.

(60) En el segundo asalto ambos luchadores combinaron striking con mucho trabajo de suelo (La Provincia, 31/03/2019).

Subprime es un anglicismo proveniente de Estados Unidos que se emplea a menudo para referirse a un tipo de hipoteca considerada "por debajo de lo óptimo".

(61) El último trimestre de ese año, cuando estalló la crisis con aquel primer capítulo de las hipotecas subprime o basura en los Estados Unidos, en las Islas había 39.500 trabajadores juveniles más de los que hay en estos momentos (La Provincia, 09/04/2019).

Styler es un término que se emplea en inglés, pese a no estar recogido en los diccionarios consultados (OED, CED y DLE). Parece ser el nombre de un modelo concreto de plancha de ropa a vapor hecho por LG. En español, se usa con el mismo significado, así que en este caso tenemos un anglicismo que ha pasado de ser un nombre propio de un producto a un nombre genérico para referirse a cualquier plancha de este estilo. Un caso similar a este, aunque no presente en este estudio, es el de Kleenex, empleado para referirse a cualquier pañuelo de papel.

(62) Esta herramienta de calor, la plancha o styler, que vive hoy sus mejores días en un mercado que ofrece las mil y una opciones, consigue varios efectos que buscan muchas mujeres.

Swag es un adjetivo para referirse a un tipo de corte de pelo que parte de una media melena al hombro con capas desestructuradas ${ }^{22}$.

\footnotetext{
21 En https://es.wikipedia.org/wiki/ [Consulta: 21/06/2020].

22 En https://www.telva.com/belleza/pelo/2019/05/24/ [Consulta: 21/06/2020].
} 
(63) En esto, el corte swag es el rey (La Provincia, 29/03/2019).

Swap es un acuerdo de intercambio financiero en el que una de las partes se compromete a pagar con una cierta periodicidad una serie de flujos monetarios a cambio de recibir otra serie de flujos de la otra parte. Estos flujos responden normalmente a un pago de intereses sobre el nominal del swap ${ }^{23}$.

(64) El juez falla contra el Santander por los 'contratos swaps' de Gáldar (La Provincia, 23/04/2019).

Letra $\mathrm{T}=19$

Tablet, tag, talent show, tattoo, teaming, threading, tie break, tightlining, tip, top, top coat, top ten, trail, trail runner, training manager, trending topic, truck food, tweed, twist.

Un talent show es un programa o concurso donde cada participante muestra cuál es su talento, especialmente en el campo del entretenimiento.

(65) Ahora, ha decidido viajar al otro lado del charco para demostrar potencial en el 'talent show' del país azteca (La Provincia, 21/03/2019).

Threading es, tal como indica el siguiente ejemplo, depilación con hilo.

(66) La depilación con hilo, o threading, es una técnica ancestral muy popular procedente de La India (La Provincia, 07/03/2019).

Tie break es un desempate en algunos deportes como el tenis y el béisbol.

(67) Triunfo sufrido del Vecindario en el 'tie break' ante el Barça (La Provincia, 17/03/2019).

Tightlining es un delineado invisible que se aplica en la línea de agua del párpado superior y entre las pestañas para dar más definición al ojo sin necesidad de llevar "eyeliner."

${ }^{23}$ En https://www.bbva.com/es/swaps-que-son-y-como-funcionan/ [Consulta: 22/06/2020]. 
(68) Habrás oído mil veces que esta raya pintada por dentro del ojo hace el ojo más pequeño. Pues bien, no es verdad. Sobre todo, si echas mano de un truco muy pro: el llamado tightlining (La Provincia, 26/04/2019).

El anglicismo top aparece recogido en el DLE, pero no con la acepción presente en el siguiente ejemplo, donde se alude a algo que ocupa los lugares más altos o mejor valorados.

(69) Las más votadas o las que aparecen en el top del ranking suelen ser seguras (La Provincia, 10/03/2019).

Trail y trail runner son dos anglicismos que hacen referencia al deporte y deportistas que corren campo a través.

(70) Para correr por caminos de tierra, por el campo o por zonas pedregosas, zapatillas de trail -que tienen mayor agarre y estabilidad- ( $\mathrm{La}$ Provincia, 08/03/2019).

Training manager es un director de formación.

(71) (Gloria Montero, Training Manager de Revitalash) (La Provincia, 23/05/2019).

Trending topic alude al tema del momento. Muy empleado en las redes sociales.

(72) El concurso de murgas logró ser sus cuatro noches de celebración trending topic nacional (La Provincia, 15/03/2019).

Tweed es un tejido elaborado a partir de la mezcla de lanas.

(73) Sus tejidos de 'tweed', ante y polipiel las hacen poco adecuadas para verano, pero ideales para cualquier otra temporada, incluso invierno (La Provincia, 20/05/2019).

Letra $\mathrm{U}=4$

Ugly shoes, ugly sneakers, underground, urban style.

Ugly shoes y ugly sneakers son dos tipos de calzado caracterizados por una enorme suela y un aspecto rudo.

(74) Así ocurrió con los 'ugly shoes' y con las 'chunky sneakers', que tuvieron detractores a puñados pero que, finalmente, terminaron 
por gustar y se hicieron un hueco en el armario de aquellas que en principio juraron odiarlas (La Provincia, 06/05/2019).

Urban style es un estilo que está constantemente reinventándose y cuya inspiración es la fusión de diferentes culturas y estilos de vida, floreciendo en las grandes ciudades ${ }^{24}$.

(75) Un ecosistema donde mezclarse, que crea sinergias para conocer, descubrir y vivir auténticas experiencias urban style (La Provincia, 23/04/2019).

Letra $\mathrm{V}=2$

Value4Money and vending.

(76) Value4money o Value for money hace referencia a la relación entre la calidad y el precio de un producto.

Letra $\mathrm{W}=6$

Wakeboard, warning, waterproof, wellness, wild blond, wood-aged beer.

Wakeboard es esquí acuático.

(77) Julia Castro, la deportista profesional de Kitesurf y Wakeboard, natural del pueblo de Morro Jable, volverá a llevar consigo el nombre, marca e imagen del municipio de Pájara (La Provincia, 31/03/2019).

Wild blond es una forma de aplicar unos reflejos de tinte rubio al cabello con una esponja.

(78) Wild blond, reflejos con esponja (La Provincia, 09/04/2019).

Wood-aged beer es cerveza envejecida en madera.

(79) El producto de Jaira logra la medalla de bronce en la categoría WoodAged Beer en la ciudad condal (La Provincia, 17/03/2019).

Letra $\mathrm{Y}=1$

Youtuber es una persona que hace y sigue vídeos en Youtube.

${ }^{24}$ En https://www.atlanticfix.co/colecciones/urban [Consulta: 22/06/2020]. 
Letra $Z=1$

Zero, que en español es "cero".

(80) Como alternativa, muchos hombres y mujeres optan por consumir refrescos light o zero con la conciencia tranquila, pensando que están bebiendo algo más sano y que engorda menos (La Provincia, 05/04/2019).

Es obvio que el porcentaje mayor de términos anglicados hallados en el análisis de la prensa empieza por la letra S, con 55 ejemplos. También las letras C, B y P son bastante prolíficas, con una media de unos 30 ejemplos por letra. En el extremo opuesto están J y X, pues no se encontró ningún anglicismo que empezara por esas letras; asimismo, Y, Z, U, V, I y K cuentan con menos de 5 préstamos cada una.

\subsubsection{Anglicismos adaptados $=23$ casos}

En esta sección se han compilado aquellas unidades léxicas anglicadas que presentan algún tipo de adaptación. La mayoría de los casos son adaptaciones ortotipográficas al español como, por ejemplo, voleibol del inglés volleyball.

A continuación, se relacionan los casos encontrados:

Antiaging / anti-aging, que provienen del término inglés antiageing. En ambos casos la $e$ de age desaparece en el español.

Buggie, que proviene del inglés buggy. La y final que se usa en el inglés pasa a ser ie, por influjo de la forma plural del inglés buggies. Exactamente lo mismo ocurre con celebritie, que en inglés es celebrity (singular), pero celebrities en plural.

Clúster, que proviene del inglés cluster, sin tilde. En español se le ha añadido la tilde a la $u$, y hace referencia a un grupo o equipo en el contexto examinado:

(81) Víctor Lugo Jorge, nuevo presidente del Clúster de Enoturismo de Canarias (La Provincia, 01/03/2019).

Hackaton $\sin b$ intercalada, que proviene del inglés hackathon. Es un término informal que aparece recogido en el OED para referirse a un evento de duración en torno a los dos o tres días en el que 
un elevado número de personas se reúnen para trabajar de forma colaborativa en programación informática.

(82) “El hackaton es una oportunidad maravillosa para nosotros" ( $L a$ Provincia, 26/03/2019).

Cow boy, separado en dos vocablos, proviene del inglés cowboy. Un caso similar es el de on line, del inglés online. Opuesto es el caso de Goalaverage, que en español es un único término, pese a que procede del inglés goal average, que son dos vocablos. Otros casos son los de realfooder (frente al inglés real fooder), o bien realfooding (del inglés real fooding). Estos términos hacen referencia a un movimiento y estilo de vida que se basa en consumir comida real o saludable y evitar productos ultraprocesados ${ }^{25}$.

(83) Carlos Ríos, dietista nutricionista, creador del movimiento en redes "realfooding" (La Provincia, 24/04/2019).

Startup, sin guion, que proviene del inglés start-up; y esports, del inglés e-sports.

Instagramer con una sola $m$,que proviene del inglés instagrammer. Similares son los casos de millenial con una sola $n$ (frente al inglés que emplea dos, millennial) y de palet con una sola $l$ (en contraste con la forma inglesa pallet). Otro ejemplo es el de skimers (del inglés skimmers, con doble $m$ ), el cual se refiere a la boca de succión instalada en las paredes de una piscina o barco, en un nivel cercano a la superficie de la misma, y que permite el correcto filtrado del agua ${ }^{26}$.

(84) skimers para la limpieza de combustible en superficie (La Provincia, 15/05/2019).

Otros casos en los que se da una adaptación a la ortografía del español son parquin, del inglés parking; smokey eye, del inglés smoky eye; tabloide, del inglés tabloid; vermut, del inglés vermouth;

\footnotetext{
En https://realfooding.com/ [Consulta: 22/06/2020].

26 En https://www.construmatica.com/ [Consulta: 22/06/2020].
} 
fast ferri, del inglés fast ferry; tuit, del inglés tweet; e, incluso, el caso de tuitera, que es un derivativo de tuit.

Como se puede observar, en la mayoría de los casos analizados se dan adaptaciones de carácter ortotipográfico y morfológico de los términos. Con respecto al nivel semántico, el significado de estas unidades léxicas anglicadas se identifica o es muy cercano al significado en la lengua origen, el inglés en este caso.

\subsubsection{Anglicismos Híbridos $=8$ casos}

En este epígrafe se recogen aquellos casos de términos combinados que unen una parte del inglés y otra parte de otra lengua. Pulcini, Furiassi y Rodríguez González los definen como "a multi-word unit which freely combines an English element with a RL element" (2012: 7).

Eservicios combina $E$ de electronic con servicios y se usa para designar a los servicios electrónicos ofrecidos por algunas administraciones públicas y privadas.

(85) La primera se desarrolló en el Hotel Escuela Esquinzo, y en ella se trabajaron temáticas como la e-Inclusión, las competencias digitales, el envejecimiento activo, los eServicios (La Provincia, 22/03/2019).

Fake relato es un término bastante usado en prensa con motivo del independentismo catalán en boca de Dolors Montserrat y, por analogía con las fake news, fake relato hace referencia a una historia inventada. En inglés el término es fake newes, por lo que se trata de una unidad léxica de nueva creación en español.

(86) Montserrat quiere frenar el "fake relato" del independentismo catalán (La Provincia, 01/04/2019).

Kitesurfista es la persona que practica el kitesurf, deporte que consiste en navegar sobre una tabla que es traccionada por una cometa. Esta combinación permite alcanzar grandes velocidades sobre el agua y realizar espectaculares saltos ${ }^{27}$. Es un vocablo que proviene del inglés kitesurfer.

27 En https://holaola.surf/tour/ [Consulta: 22/06/2020]. 
(87) La kitersurfista Julia Castro, imagen deportiva del municipio ( $L a$ Provincia, 31/03/2019).

Salvaslips combina el español salva con el término inglés slips. Los salvaslips sonuna especie de compresa más fina y pequeña y con mucho menos poder de absorción. El término empleado en inglés es panty liner, por lo que el vocablo en cuestión es una creación del español.

(88) Útiles de higiene personal como tampones, compresas, pañuelos de papel, toallitas húmedas, salvaslips, algodones [...] organizados y guardaditos (La Provincia, 05/04/2019).

Shopping virtual se refiere a la venta de productos vía internet a través de portales web. En este caso, observamos cómo estos dos anglicismos se rigen por el orden normal del español (sustantivo + adjetivo) y no el del inglés, en el que aparecería antes el adjetivo, tal como virtual shopping.

(89) el informe añade que es "muy probable" que ese usuario esté informándose de futuras compras, tal como hace un $22 \%$ de canarios, que entre las 18.00 y las 22.00 horas se van de 'shopping virtual', el llamado nuevo prime time del consumo (La Provincia, 15/04/2019).

Eco green combina la raíz del español ecológico con el vocablo inglés green. Se trata de un término que hace referencia a un modelo de acción tanto por parte de empresas como de individuos donde predominan la sostenibilidad y las tres $\mathrm{R}$ : reciclar, reutilizar y reducir.

(90) Lo ecológico y lo natural cada vez se están abriendo más paso en nuestras vidas. Pero no sólo a la hora de llevar una vida sana o contaminar menos: este movimiento 'eco green' también ha llegado a nuestras casas (La Provincia, 17/05/2019).

Voz en off hace referencia a una técnica de producción donde se retransmite la voz de un individuo que no está visualmente delante de la cámara durante una producción de televisión ${ }^{28}$.

28 En https://es.wikipedia.org/wiki/ [Consulta: 22/06/2020]. 
(91) Imitando la dinámica del concurso 'Un, Dos, Tres. Responda otra Vez', una voz en off realiza una serie de preguntas sobre actualidad (La Provincia, 28/03/2019).

Plogger / plogging es una moda nacida en Suecia que consiste en recolectar basura a la par que se corre o trota. En este caso particular, se combina la raíz del verbo sueco para "recoger" con el inglés jogging.

(92) Cuentan en Instagram los ploggers habituales que llegan a recoger kilos de basura en cada salida (La Provincia, 10/04/2019).

(93) Apúntate a practicar plogging, la versión más ecológica del running (La Provincia, 10/04/2019).

En líneas generales, en el caso de los anglicismos híbridos, se puede observar la "traducción” de alguno de los elementos, pero también la formación de nuevas unidades léxicas a partir de elementos de procedencia inglesa combinados con elementos del español.

\subsubsection{Falsos Anglicismos $=4$ casos}

Esta sección recoge aquellos términos que, pese a que tienen la apariencia del inglés, no existen con el mismo significado en la lengua inglesa. Pulcini, Furiassi y Rodríguez González los definen como "a word or multi-word unit in the RL made up of English lexical elements but unknown or used with a conspicuously different meaning in English” (2012: 7). A este respecto, Campos Pardillos afirma que

if a language is ready to use an English word because of prestige, conciseness or a desire to put on an appearance of expertness, or in other words, if it is willing to resort to "imported goods," it might also feel inclined to "copy" or "imitate" the English language. This may be the case of the so-called "false Anglicisms" (2015: 158).

Tales son los casos de:

Anti-balconing, que se refiere a una campaña que va en contra de la práctica del balconing, actividad que consiste en lanzarse a una piscina desde el balcón de un apartamento, con los consiguientes riesgos que conlleva. 
(94) Una joven británica inicia una campaña 'antibalconing' (La Provincia, 27/03/2019).

Bunkering designa al abastecimiento y repostaje de los buques en el mar. Se realiza prácticamente a diario a través de un buquetanque o gabarra que se pega al costado del barco que va a repostar, al que traspasa el combustible a través de una serie de bombas ${ }^{29}$.

(95) El bunkering se une en torno a una comisión de Fedeport (La Provincia, 08/03/2019).

Terrazing hace referencia a una campaña que tiene por objeto concienciar a la población española sobre los peligros de exponerse al sol sin protección ${ }^{30}$.

(96) El Grupo Español de Pacientes con Cáncer (GEPAC) ha lanzado, con motivo del Día Mundial del Cáncer de Piel y Melanoma, la campaña 'terrazing', que tiene como objetivo concienciar a la población española sobre los peligros de exponerse al sol sin protección, ya sea en la playa, en el campo, esquiando, en un partido de fútbol o tomando un refresco con amigos en una terraza (La Provincia, 28/05/2019)

Eyecat es un neologismo que se emplea en el ámbito de la belleza para referirse a una forma de maquillarse los ojos, así como a un tipo de esmaltado de uñas. En inglés se emplea cat eye, pero no eyecat; de ahí que este último sea considerado un falso anglicismo.

(97) En plan eyecat grueso, en azul cobalto con un toque metálico, como en el desfile de primavera-verano de Gabriela Hearst (La Provincia, 03/04/2019).

3.2. Funciones de los anglicismos en la prensa analizada

Las razones que pueden explicar o justificar los usos de estos anglicismos en la prensa analizada son variadas. En este epígrafe haremos referencia a algunas de ellas.

29 En https://www.larazon.es/ [Consulta: 22/06/2020].

30 En http://cancerpiel-melanoma.com [Consulta: 23/06/2020]. 
En primer lugar, como es bien sabido, el inglés se asocia a valores como lo moderno. Por tanto, en casos como los de Local Craft Beer, science show y trending topic, cabe considerar el deseo por parte del redactor o periodista de parecer sofisticado o profesional. Tales vocablos pueden expresarse en español como "cerveza artesanal local”, "espectáculo o programa de ciencia” y "tema actual”, no obstante, se escoge la opción de expresarse en inglés. Por consiguiente, el uso de estos extranjerismos parece estar asociado con cierto esnobismo.

También muchos de estos anglicismos forman parte de un lenguaje de especialización dentro de un campo determinado, como puede ser el del deporte, la economía o la moda. Se trata de unidades léxicas que se refieren a conceptos o realidades concretas (ver definiciones y usos en epígrafe 3.1.1.) para las cuales no existe equivalente en español. Algunos ejemplos son grappling, scratch, shakedown, multistakeholder, tightlining, striking y threading.

Algunas de las lexías anglicadas extraídas desempeñan una función referencial que consiste en el empleo de un anglicismo para designar conceptos que surgen en la cultura anglonorteamericana y se exportan a la cultura de la lengua receptora, como pueden ser los casos de baby shower o batch cooking. Son conceptos relativamente novedosos que provienen del mundo anglonorteamericano y se instalan en la cultura latina con el nombre original inglés.

Otros préstamos son empleados en la prensa con una función expresiva, con fines eufemísticos o el deseo de evitar usar un vocablo que podría resultar malsonante en español. Tal es el caso de tattoo, que tal vez atenúe al de la voz tatuaje, término que en español aún podría considerarse cargado de connotaciones peyorativas en determinados contextos.

La función textual o de economía del lenguaje puede también justificar algunas de estas lexías anglicadas. Un ejemplo es manspreading, que denomina la práctica de los hombres que se sientan con las piernas abiertas en el transporte público, ocupando así más de un asiento y pudiendo incomodar a otros usuarios. Otro caso es el del neologismo phubbing, combinación de los términos phone y 
snubbing (desprecio, desaire), que consiste en menospreciar a quien nos acompaña al prestar más atención al móvil u otros aparatos electrónicos que a su persona. No solo no existe un término equivalente en español para referirse a estos conceptos, sino que además se ha de usar una larga definición para describir su significado.

\subsection{Comparativa entre el estudio publicado en 1999 y este estudio}

El aumento en el empleo de anglicismos también se pone de relieve al comparar los resultados encontrados en 1997, pese a que se publicó en 1999 (Luján-García 1999) y este estudio. En el primero se encontraron un total de 355 anglicismos en el análisis de los titulares del mismo periódico, La Provincia, a lo largo de todo el año 1999. El presente trabajo desvela la presencia de 313 anglicismos en el seguimiento de las noticias de tan solo tres meses del año 2019 (marzo, abril y mayo). Si bien el estudio de 1999 se centró en el análisis de los titulares periodísticos y el presente trabajo se centró en el análisis de toda la noticia, debemos señalar que el incremento de anglicismos ha sido exponencial. Además, se puede observar cómo muchos de los anglicismos presentes en el estudio de 1999 ya están recogidos y normalizados en el $D L E$ y, por eso, no han sido incluidos en el presente estudio. Tal es el caso de vocablos como chip, funky, handicap, hacker, hip hop, bockey, jazz, souvernir, sexy, show, surf y western, por mencionar algunos ejemplos.

Por consiguiente, podemos considerar que no solo se utiliza mayor cantidad de anglicismos en la prensa analizada actual, sino que además se trata de anglicismos novedosos que reflejan más la actualidad, tales como startup, batch cooking y phishing. Muchos de estos anglicismos no aparecían en el estudio de 1999, ya que estos conceptos no estaban de moda o simplemente no existían hace dos décadas. 
3.4. Marcadores ortotipográficos empleados para señalar que son extranjerismos

Conviene señalar que no parece haber consistencia en lo que se refiere a los símbolos ortográficos que especifican que se trata de extranjerismos. Como se refleja en la tabla 2, en la mitad de los casos encontrados el anglicismo aparece sin ningún tipo de marcador. En un porcentaje inferior, las unidades léxicas analizadas aparecen entre comillas simples, con un $23,4 \%$; seguidas por el uso de la cursiva, con un $18,9 \%$ y en porcentajes bastante inferiores se encuentra el empleo de las comillas dobles, con un 3,3\%, y la negrita, con un $2,8 \%$. En algunos casos aislados se emplean otros marcadores como la combinación de las comillas simples y la negrita $(1,5 \%)$, la mayúscula inicial $(0,05 \%)$ y las comillas simples y cursiva $(0,02 \%)$.

Tabla 2. Uso de marcadores para señalar los Anglicismos

\begin{tabular}{lcc}
\hline & MARCADORES & $\%$ \\
\hline SIN NINGÚN MARCADOR & 173 & $50 \%$ \\
COMILLAS SIMPLES & 80 & $23,2 \%$ \\
COMILLAS SIMPLES + NEGRITA & 5 & $1,5 \%$ \\
COMILLAS SIMPLES+CURSIVA & 1 & $0,02 \%$ \\
COMILLAS DOBLES & 11 & $3,2 \%$ \\
CURSIVA & 65 & $18,7 \%$ \\
NEGRITA & 9 & $2,6 \%$ \\
MAYÚSCULA INICIAL & 2 & $0,05 \%$ \\
TOTAL & 346 & \\
\hline
\end{tabular}

El Diccionario Panbispánico de Dudas (DPD), en su tratamiento de los extranjerismos, señala que se debe recurrir al empleo de la cursiva o las comillas para marcar que se trata de un vocablo ajeno al español. Sin embargo, como reflejan los resultados de este análisis, no siempre se respetan estas recomendaciones. 


\section{Conclusiones}

Pese a sus posibles limitaciones, el presente trabajo ofrece evidencias de una notable presencia de términos anglicados en la prensa digital analizada. Con respecto al tipo de anglicismo usado mayormente, este es, sin duda alguna, el anglicismo no adaptado (con casi un $89,3 \%$ del total); seguido muy de lejos por los anglicismos adaptados (con un $7 \%$ ) y los híbridos (2,4\%); por último, los falsos anglicismos $(1,2 \%)$ tienen una representación simbólica dentro del corpus compilado. Este resultado va en consonancia con el de otros trabajos recientes que ponen de manifiesto que la tendencia gira en torno al uso de anglicismos puros o sin ningún tipo de adaptación en cualquier terreno del español.

Un trabajo anterior publicado hace ya varias décadas (LujánGarcía 1999) analizaba el empleo de anglicismos en el mismo diario, La Provincia, y los resultados arrojaron una presencia de 355 anglicismos en los titulares de las noticias publicadas a lo largo de todo el año 1999. Dos décadas más tarde podemos observar un aumento exponencial en la cantidad y variedad de anglicismos, pues en un seguimiento de solo tres meses (marzo, abril y mayo de 2019) se han hallado un total de 332 anglicismos, mientras que en el estudio de 1999 no se reconoce la presencia de anglicismos híbridos o falsos anglicismos.

Como se describe en las líneas anteriores, no existe una única razón para el empleo de estos extranjerismos en español y la realidad es que la tendencia sigue al alza; son cada vez más los términos anglicados empleados en la prensa digital.

En relación con el empleo de marcadores ortotipográficos, el $50 \%$ de los anglicismos se emplea sin ningún tipo de marcador, lo cual hace que no se siga la recomendación de la DPD a este respecto. Con porcentajes inferiores se emplean las comillas simples o la cursiva.

La percepción y grado de comprensión de estos anglicismos por parte de los lectores podría ser objeto de un análisis futuro. En cualquier caso, nos atrevemos a vaticinar que, conforme el nivel de 
inglés va ascendiendo entre las generaciones más jóvenes, también parece existir una mayor comprensión, normalización e, incluso, uso de estos préstamos en español.

\section{Referencias bibliográficas}

Balteiro, Isabel y Miguel Ángel Campos

2012 "False anglicisms in the Spanish language of fashion and beauty". Ibérica. 24, 233-260.

Bolaños-Medina, Alicia y Carmen Luján-García

2010 "Análisis de los anglicismos informáticos crudos del léxico disponible de los estudiantes universitarios de traducción”. Lexis. 34, 2, 241-274.

Cambridge English Dictionary (CED). Consultado: 9 de julio de 2019 https://dictionary.cambridge.org/es/.

Campos Pardillos, Miguel Ángel

2015 "All is not English that Glitters: False Anglicisms in the Spanish Language of Sports.” Atlantis. 37, 2, 155-174.

Crespo-Fernández, Eliecer y Carmen Luján-García

2013 "Anglicismo y tabú: valores axiológicos del anglicismo". Revista de Estudios Filológicos. 52, 53-74.

Crespo-Fernández, Eliecer y Carmen Luján-García

2017 "Anglicisms and Word Axiology in Homosexual Language". Revista Española de Lingüística Aplicada (RESLA). 30, 1, 74-102. <https://doi.org/10.1075/resla.30.1.04cre>

Crespo-Fernández, Eliecer y Carmen Luján-García

2018 Anglicismos sexuales en español. El inglés como recurso enfemístico y disfemístico en la comunicación virtual. Editorial Comares: Granada.

Esteban AsEncio, Laura

2008 "Uso, origen y procesos de creación de neologismos en prensa española”. Círculo de lingüística aplicada a la comunicación. $33,3-27$. 
García-Morales, Goretti; Isabel GonZÁlez-Cruz; Carmen Luján-GarCíA; y María Jesús RodrígueZ-MedinA

2016 La presencia del inglés en la publicidad televisiva española (2013-2015). Madrid: Síntesis.

Gerding, Constanza; Paola Cañete; y Carolin Adam

2018 "Los neologismos por préstamo y los periodistas: cuestionario". REDILLET. 1.

LÓPEZ-Zurita, Paloma

2005 "Economic anglicisms: Adaptation to the Spanish linguistic system”. Ibérica. 10, 91-114.

Luján-García, Carmen

1999 "Anglicismos en los titulares de la prensa canaria actual: Un estudio comparativo”. Philologica Canariensia. 4-5, 129-146.

LujÁN-García, Carmen

2011 "English 'invasion' in Spain: An analysis of toys leaflets addressed to young children”. English Today. 3, 3-9.

LujÁN-García, Carmen

2015 "Lexical anglicisms: An analysis of commercials addressed to young children on four Television Channels in Spain”. Onomazein. 32, 288-304.

LujÁn-García, Carmen

2017a "Analysis of the presence of Anglicisms in a Spanish internet forum: some terms from the fields of fashion, beauty and leisure". RAEI. 30, 277-300.

LujÁN-García, Carmen

2017b "Anglicisms and their use in an Internet Forum Adressed to Spanish- speaking Teenagers". Spanish in Context. 14, 3, 440463. <https://doi.org/10.1075/sic.14.3.05luj>

LujÁN-GARCía, Carmen (ed.)

2021 Anglicismos en los nuevos medios de comunicación. Tendencias actuales. Granada: Comares.

Oxford English Dictionary (OED). Consultado: 10 de julio de 2019. $<$ https://www.oed.com/> 
PAno, Ana

2007 "Los anglicismos en el lenguaje de la informática en español. El 'misterioso mundo del tecnicismo' a través del tecnicismo a través de foros y glosarios en línea”. Bolonia: Centro di Studi Lingüístico-Culturali (CeSLIiC), p. 18. <https://doi. org/10.6092/unibo/amsacta/2370>

Pulcini, Virginia; Cristiano Furiassi; y Félix Rodríguez GonZÁlez 2012 "The lexical influence of English on European languages: From words to phraseology". En The Anglicization of European Lexis. Eds., Cristiano Furiassi, Virginia Pulcini y Félix Rodríguez González. Amsterdam/Philadelphia: John Benjamins Publishing, 1-24.

Real Academia Española (RAE)

2005 Diccionario Panbispánico de dudas. Real Academia Española: Madrid.

Real Academia Española (RAE)

Diccionario de la Lengua Española (DLE). Consultado: 6 de julio de 2019. <https://dle.rae.es/>

Raidel Naranjo, Carlos

2017 "Transferencia léxica de los anglicismos en la prensa santiaguera (1805-1902). Un estudio sociolingüístico histórico”. Disertación inédita. Doctor en filosofía. Facultad del Departamento de Estudios Hispánicos de la Universidad de Houston.

Rodríguez DíAz, José Antonio

2011 "Anglicismos y germanismos en el lenguaje de la publicidad del español peninsular contemporáneo 1998-2007”. Tesis doctoral inédita. Universidad de Santiago de Compostela.

Rodríguez GonZÁlez, Félix

2012 "Anglicismos en el mundo del deporte: variación lingüística y sociolingüística”. Boletín de la Real Academia Española (BRAE). CXII (XXXVI), 261-285.

RODRíGUEZ GONZÁLEZ, Félix

2016 "Usos metafóricos de anglicismos en el lenguaje futbolístico". Puntoycoma. 146, 34-39.

Rodríguez GonZÁlez, Félix

2017 Gran Diccionario de Anglicismos. Madrid: Arco Libros. 
Rodríguez GonZÁlez, María Jesús

2016 "An approach to the study of the use of English in the Activities of Spanish Gyms". Spanish in Context. 13, 1, 128148. <https://doi.org/10.1075/sic.13.1.06rod>

RodrígueZ-Medina, María Jesús

2021 "Este jugador está en el top ten de los cracks: anglicismos en la prensa deportiva en las redes.” En Anglicismos en los nuevos medios de comunicación. Tendencias actuales. Ed., Carmen Luján-García. Granada: Comares, 121-140.

Tejedor Martínez, Cristina

2017 "The influence of the English language on the description of cosmetic products". Revista Alicantina de Estudios Ingleses. 30, 303-329.

Tejedor Martínez, Cristina

2021 "El look de las celebrities o la imagen de los famosos. La influencia del inglés analizada a través de las secciones sobre gente y estilo en la prensa." En Anglicismos en los nuevos medios de comunicación. Tendencias actuales. Ed., Carmen Luján-García. Granada: Comares, 45-72.

VÁzquez Amador, María y Carmen Lario de Oñate

2015 "Anglicismos en la prensa deportiva de principios del siglo XX y XXI: estudio contrastivo”. EPOS. 31, 359-374.

VÁzQuez Amador, María

2018 "Los anglicismos de la moda en la prensa rosa española”. Onomazein. 40, 49-55.

Vega Falcón, Nadia

2017 "Anglicismos en prensa de moda internacional y su uso a través de las TIC por parte de estudiantes universitarios”. Tesis doctoral inédita. Universidad de Las Palmas de Gran Canaria

Vélez BARReiro, Marco

2003 “Anglicismos en la prensa económica”. Tesis doctoral inédita. Universidade da Coruña. 